\title{
COMPARATIVE EVALUATION OF THE MASTICATORY MUSCLES ACTIVITY WITH CAD/CAM VERSUS CONVENTIONAL COMPLETE DENTURE USING ELECTROMYOGRAPHY
}

\author{
Mahmoud Darwish Mohamed* and Nasser Hussein Shaheen**
}

\begin{abstract}
Purpose: To evaluate in-vivo the effect of CAD/CAM constructed dentures on the masticatory muscles activity using electromyography in comparison to conventionally constructed dentures.

Materials and Methods: Fourteen completely edentulous patients were randomly allocated to two equal groups. Patients in the first group received conventionally constructed complete dentures and patients in the second group received complete dentures constructed using CAD/CAM technology. After 30 days of denture use the anterior temporalis and superficial masseter muscles activity were measured by EMG. A cross over was performed for patients with conventional denture to $\mathrm{CAD} / \mathrm{CAM}$ and vice versa and the dentures were used for further 30 days, after which measurements of anterior temporalis and superficial masseter muscles activity were taken using EMG. Electromyographic data was recorded and analyzed, mean values were compared by independent $t$-test to compare between the two groups as regard to muscle activity.
\end{abstract}

Results: There was a significant difference in the activity of the masseter muscles and the anterior temporalis muscles for the patients received CAD/CAM denture in comparison to those received the conventional denture, after 30 days of denture use $(p<0.05)$.

Conclusion: CAD/CAM constructed complete dentures produced higher muscle activity if compared to conventionally constructed complete dentures.

\section{INTRODUCTION}

Construction of removable complete dentures considered the least invasive as well as most cost effective option for the rehabilitation of completely edentulous patients ${ }^{(1)}$. The main purpose of complete denture treatment is to restore masticatory function, facial aesthetic, and enhance patients' confidence, and the use of full dentures helps edentulous patients obtain adequate nutrition (2). However, complete denture has some limitations since the masticatory efficiency of denture wearers is less than of dentate patients ${ }^{(3)}$. For effective mastication, stability,

* Lecturer of Removable Prosthodontics, Faculty of Dentistry, Suez Canal University, Ismailia, Egypt.

** Associate Professor, Department of Removable Prosthodontics, College of Oral and Dental Surgery, Misr University for Science and Technology, Egypt. 
retention and support principles in complete dentures must be considered.

For proper diagnosis and treatment planning of edentulous patients, the functional behavior of masticatory muscles should be properly understood and analyzed using objective and subjective methods ${ }^{(4)}$. Masticatory muscle behavior can be objectively evaluated during function using electromyographic activity (EMG) by capturing electric potentials from the masticatory muscles during rest and function. Surface electromyography is used to measure the muscular activity noninvasively using surface electrodes placed on the skin overlying the muscle ${ }^{(5)}$.

Currently, complete dentures that planned and constructed using conventional methods requires a broad series of laboratory and clinical procedures ${ }^{(6)}$. Edentulous patients have to visit the dental clinics five or more times, including diagnosis, primary impressions, final impressions, recording of maxillo-mandibular relationship, trial placement of the waxed-up denture, and insertion of the denture. All these laboratory and clinical procedures are performed manually. Therefore, it is very difficult to ensure the quality for these manually planned and constructed dentures.

Computer-aided design and computer-aided manufacturing (CAD/CAM) has emerged as a new approach for the design and fabrication of complete dentures, the manufacturing process is subtractive, the denture bases are milled from fully polymerized acrylic resin pucks ${ }^{(7)}$ and are therefore not subject to shrinkage or distortion phenomena anymore ${ }^{(8)}$. The use of CAD/CAM technology in the field of dentistry could be traced back to the early $1980 \mathrm{~s}^{(7)}$. However, in spite of more use of this CAD/CAM technology in the other aspects of dentistry, it is highly limited in the construction of complete dentures due to the lack of suitable CAD software ${ }^{(9)}$.

Recently several CAD software systems, such as AvaDent digital dentures and 3Shape Dental Sys- tem become available for designing complete edentulous dentures. Using CAD/CAM technology, only two appointments are needed for complete denture construction. Impressions, maxillo-mandibular relations registration, tooth mold and shade selection, and maxillary anterior tooth positioning could be achieved in first patient visit for constructing of the denture, saving time, materials and efforts for both dentists and patients ${ }^{(10-11)}$.

Steinmassl et. al. ${ }^{(12)}$ concluded from an in-vitro study that CAD/CAM constructed dentures have better fit than conventional dentures that explain the clinically observed enhanced retention and lower traumatic ulcer-frequency in $\mathrm{CAD} / \mathrm{CAM}$ dentures. In another study conducted by Ali and Al-Harbi ${ }^{(13)}$, they mentioned that using the CAD/CAM complete denture increased frictional retention, stability and improved its clinical performance due to good base adaptation and border seal.

Up to our knowledge there have been several studies in evaluating muscle activity using EMG with conventional complete denture ${ }^{(2,6)}$. However, none of the reports has discussed how using CAD/ CAM complete denture affect the masticatory muscle activity.

The aim of this in vivo study was to evaluate through EMG the masseter and anterior temporalis muscles activity with CAD/CAM complete denture in comparison to conventional one. The hypothesis was that, there are differences in muscle activity would be found between CAD/CAM complete denture and conventional denture.

\section{MATERIALS AND METHODS}

This study included a clinical experiment with a cross-over clinical trial design. The subjects of this study were 14 male patients in hospital clinics of Alfarabi Colleges for Dentistry and NursingRiyadh -Saudi Arabia, with mean age of 53.3 years, wearers of complete denture for 5 years at least, who met the inclusion criteria, which were edentulous 
patients who needed complete denture treatment, Angle class I jaw relation, normal alveolar ridge volume, no temporomandibular joint disorders, no heart disease, and no systemic diseases that affect the complete denture treatment procedures. Unwilling patients to participate in this study were excluded. Patients who accept to participate in this survey were received a full explanation of the study and were provided written informed consent prior to participation. The proposal of this study was reviewed and approved by the Institutional Review Board at Alfarabi Colleges for Dentistry and Nursing- Riyadh- Saudi Arabia. Electromyogram (EMG) recordings for each right and left masseter muscle and the anterior fibers of the temporalis muscle were obtained using bipolar surface EMG (Nicolet Compass Meridian 1.2.1; Natus Medical Inc. CA - USA).

Half of the subjects were randomly selected for complete denture constructed by conventional method (Group A), and the other half were selected for complete denture constructed by CAD/CAM (Group B). Both groups were instructed to use their dentures for 30 days, and on the 30th day, the anterior temporalis and superficial masseter muscles activity were measured by EMG. A cross over was performed for patients with conventional denture to $\mathrm{CAD} / \mathrm{CAM}$ and vice versa and the dentures were used for further 30 days, after which measurements of anterior temporalis and superficial masseter muscles activity were taken using EMG.

\section{Conventional Denture Construction}

Dentures for group A were constructed following the conventional clinical methods according to the guidelines of the British Society for the Study of Prosthetic Dentistry ${ }^{(14)}$. Cross linked acrylic resin teeth (Vitapan acrylic teeth, Vita, Bad Säckingen -Germany) was arranged on the center of ridge crest, using an average value articulator and balanced occlusion was achieved. The polished surface was contoured using conventional principles of waxing method.

\section{CAD/CAM denture Construction:}

Digital dentures were constructed using the AvaDent digital system (AvaDent TM Digital Dentures, Scottsdale, AZ, USA) with materials and techniques provided by the manufacturer. The AvaDent denture can be completed in 2 appointments, the measurements and records were recorded at the first appointment and the dentures inserted at the second appointment. Briefly, in the first appointment maxillary and mandibular impressions were made using provided thermoplastic trays and AvaDent heavy body and light body polyvinylsiloxane border molding and impression materials, respectively. (Fig. 1)

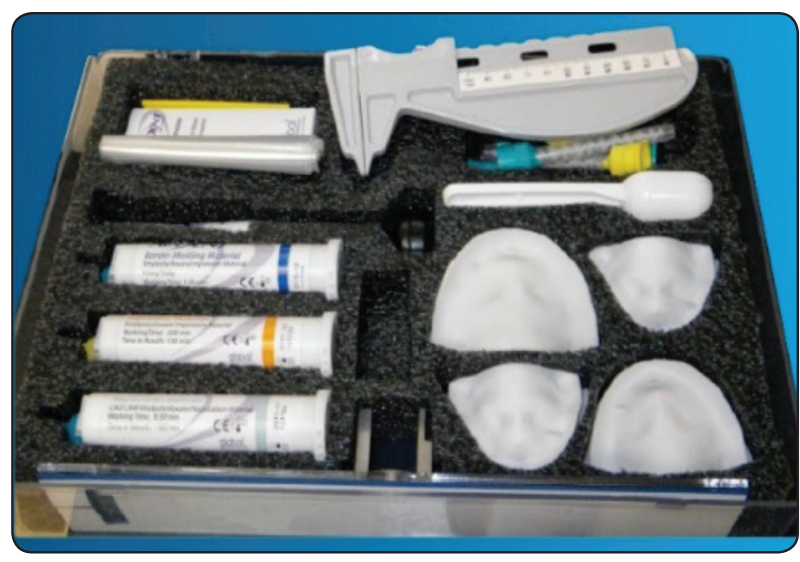

Fig. (1) The AvaDent digital system.

The maxillary-mandibular relationship was recorded using the Anatomic Measuring Device (AMD; Global Dental Science, Scottsdale, AZ, USA) that was provided (Fig. 2). AMD consists of maxillary and mandibular partial arch trays, which are available in different sizes. The mandibular AMD tray is equipped with a tracing table, and the maxillary tray has an integrated, centrally adjustable bearing pin so that a Gothic arch tracing can be made. The maxillaryAMD tray also includes an adjustable lip support flange. To stabilize the AMD on the residual ridge before making the record, the AMD maxillary and mandibular trays were 
relined with fast set polyvinylsiloxane impression material, following the determination of the vertical dimension of occlusion (VDO), the relined AMD trays are placed in the patient's mouth, and the patient is requested to close until the adjustable pin touches the tracing table. A screw driver is then used to move the central bearing pin up or down until the appropriate VDO is obtained. A Gothic arch tracing is then recorded by having the patient make protrusive and lateral mandibular movements. The apex of the Gothic arch tracing resembles an arrow and represents centric relation. A round acrylic resin bur is used to create a small depression at the apex of the arrow, the mandible is then guided until the pin fits in the created depression, and an interocclusal registration material is injected between the maxillary and mandibular AMD trays to secure them together. Flange and tooth mould templates were also used to determine lip support, midline of the lip, the horizontal lip line, and the appropriate tooth size and shape. The completed impressions and the final AMD were sent to the laboratory for fabrication of the dentures. Following the scanning of the AMD and the definitive impressions, a virtually designed complete denture was sent back for evaluation and approval of the design prior to milling of the final dentures. Following the milling of the complete denture, insertion procedures are the same as those used for any conventional complete denture.

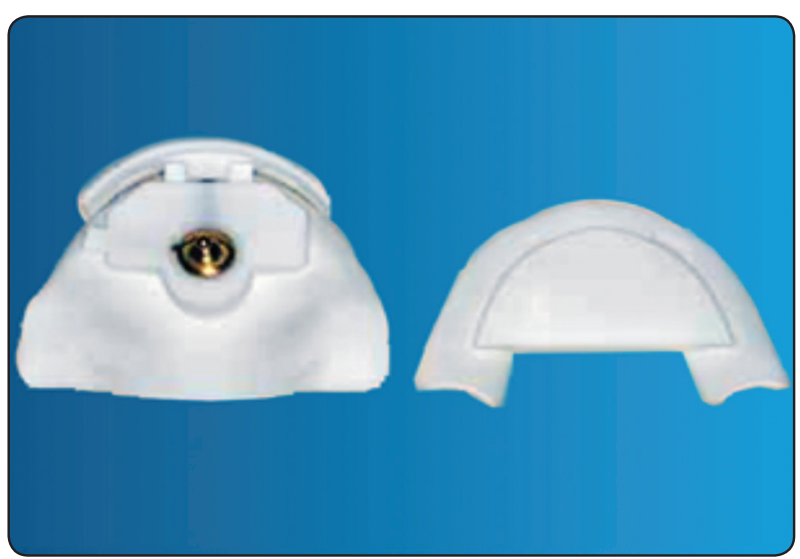

Fig. (2) Anatomic Measuring Device (AMD).

\section{Electromyographic Recording:}

After an adaptation period of 1 month the EMG recordings were made with both types of dentures. EMG recordings for each right and left masseter muscle and the anterior fibers of the temporalis muscle were obtained using bipolar surface EMG (Nicolet Compass Meridian 1.2.1; Natus Medical Inc. CA - USA). The masseter and anterior temporalis muscles on both sides were thoroughly examined by a single experienced operator at morning. The skin should properly cleaned with ethanol before electrode placement to reduce impedance. Pre-gelled, silver/silver chloride bipolar disposable electrodes were used (diameter $10 \mathrm{~mm}$, inter-electrode distance $21+1 \mathrm{~mm}$ ) ${ }^{(15)}$ and a ground electrode was attached to the right wrist. Surface electrodes were adjusted on the masseter and temporalis muscles parallel to the muscle fibers. The electrodes on the temporalis muscle were adjusted vertically along the anterior margin of the muscle. On the masseter muscles, the electrodes were adjusted parallel to muscular fibers, with the upper pole of the electrode at the intersection between the tragus-labial commissure and the exocanthion-gonion lines. During procedure, the patient sat with their head unsupported and asked to maintain a natural erect position nearly parallel to ground. Patients were instructed to bite forcefully in maximum intercuspation and to maintain the same level of contraction for 5 seconds. Three bite force recordings were made at 30 second intervals for each muscle testing. The recording was done with the same operator, and EMG data collection was made by experimenter who was blind to the subject group, and only numerical codes were used. Electromyographic data was recorded and analyzed using a fully computerized system. Each of the three recordings was analyzed for peak amplitude and duration of the longest wave and was subjected to statistical analysis. 


\section{Statistical analysis}

Data from the two groups were collected, tabulated, and statistically analyzed. The data were summarized as means and standard deviations in table 1. Collected data were analyzed using a SPSS statistical package (Version 19, Chicago, IL, USA).

Mean values were compared by independent t-test to compare between the two groups as regard to muscle activity. The five percent significance level was set for all statistical analyses.

\section{RESULTS}

The flow diagram of this clinical cross over study is given in figure 3 . Throughout the period of the study, all the involved patients completed the evaluations in the trial without drop out due to the short duration of the study.

\section{Masseter Muscle Activity:}

The comparison of the effect of conventional and CAD $\backslash C A M$ dentures on EMG activity of masseter muscles is shown in table 1. Mean and standard deviation values of the EMG activity amplitude in microvolts $(\mu \mathrm{V})$, at maximum intercuspation for conventional and CAD/CAM dentures were $(133.736 \pm 9.6236)$ and $(142.307 \pm 7.7580)$ respectively. It was observed from statistical analysis that the difference between the study groups during maximum intercuspation after 1 month of denture use was statistically significant $(P<0.05)$.

\section{Temporalis muscle activity}

The comparison of the effect of conventional and CADICAM dentures on EMG activity of Temporalis muscles is shown in table 1. Mean and standard deviation values of the EMG activity amplitude in microvolts $(\mu \mathrm{V})$, at maximum intercuspation for conventional and $\mathrm{CAD} / \mathrm{CAM}$ dentures were $(115.279 \pm 6.8306)$ and $(122.871 \pm 11.3224)$ respectively. It was observed from statistical analysis that the difference between the study groups during maximum intercuspation after 1 month of denture use was statistically significant $(P<0.05)$.

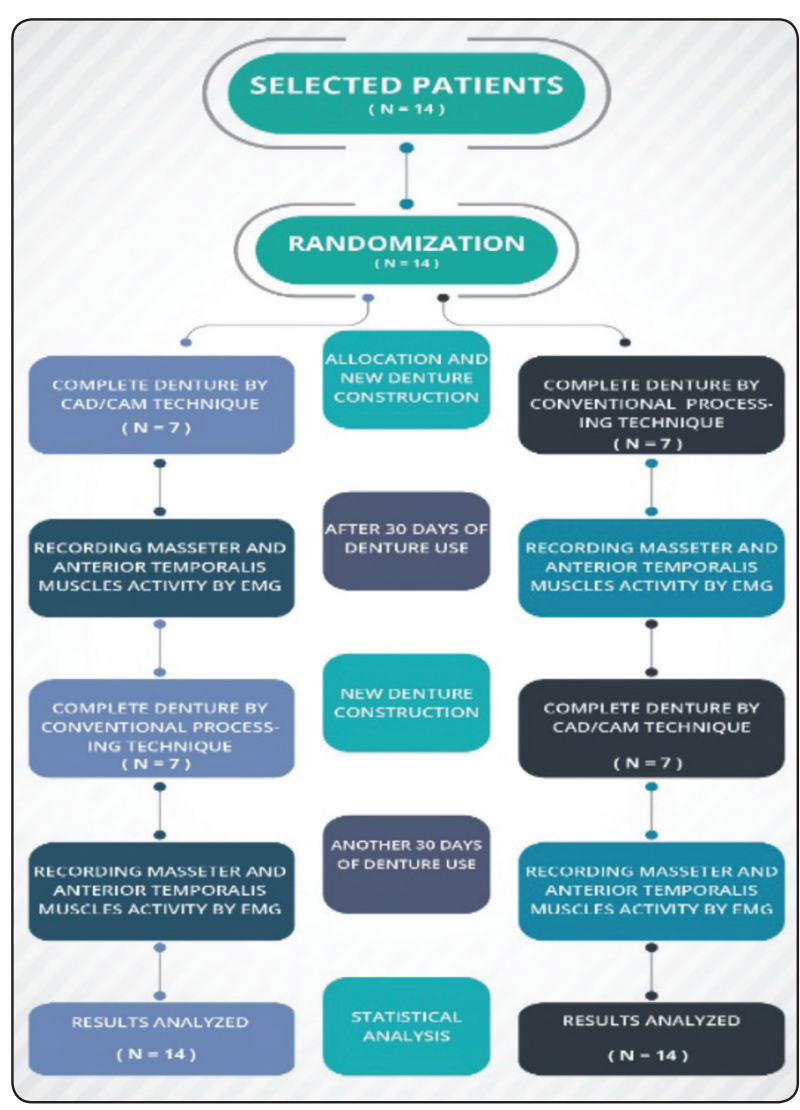

Fig. (3) The flow diagram of the study.

TABLE (1) Values of the EMG activity for Masseter Muscle and Temporalis Muscle with conventional and CAD/CAM constructed dentures.

\begin{tabular}{|l|c|c|c|c|}
\hline \multirow{2}{*}{} & \multicolumn{2}{|c|}{$\begin{array}{c}\text { Masseter Muscle } \\
\text { Activity by EMG } \\
(\mu \mathrm{V})\end{array}$} & \multicolumn{2}{|c|}{$\begin{array}{c}\text { Temporalis Muscle } \\
\text { Activity by EMG } \\
(\mu \mathrm{V})\end{array}$} \\
\cline { 2 - 5 } & Mean & SD & Mean & SD \\
\hline $\begin{array}{l}\text { Conventional } \\
\text { Denture }\end{array}$ & 133.736 & 9.6236 & 115.279 & 6.8306 \\
\hline $\begin{array}{l}\text { CAD/CAM } \\
\text { Denture }\end{array}$ & 142.307 & 7.7580 & 122.871 & 11.3224 \\
\hline P Value & \multicolumn{2}{|c|}{$0.016^{*}$} & \multicolumn{2}{|c|}{$0.043 *$} \\
\hline
\end{tabular}

SD; standard deviation. * Significant difference at 0.05 


\section{DISCUSSION}

The cross over study design used in this study reduces the between patient variability, because the comparison of denture types is made on the same patient. Moreover, this design allows the use of small sample size than parallel groups trial design. Male patients were only selected to participate in this study to avoid the difference in muscle efficiency between male and female sexes (16). Many factors may contribute to preference of male selection ${ }^{(17)}$, including hormonal alternations ${ }^{(18)}$, blood pressure ${ }^{(19)}$ and psychological factors (20). Patients with neuromuscular disorders as well as temporomandibular joint dysfunction were excluded to avoid any effect on the muscle tone, masticatory efficiency and disturbance in muscle behavior (21). Moreover; patients with abnormal ridge relationship were avoided as patients with normal ridge relationships were found to have a better masticatory efficiency than patients with abnormal one ${ }^{(22)}$. Because CAD/CAM techniques rely on the use of Gothic arch tracings to Record jaw relationship, there is a concern about certain clinical situations such as those with severe skeletal discrepancies, ridge resorption and patients with ataxia of the mandible ${ }^{(23)}$. To understand the masticatory muscles activity, electromyography (EMG) has been used. EMG is a sensitive tool used to analyze and quantify the behavior of the masticatory muscles. It has been widely used for measuring muscle function since first introduced by RE Moyers in $1949{ }^{(4)}$.The masseter and anterior temporalis muscles were evaluated as they considered as the largest and strongest of the masticatory muscles, the most superficial and are accessible to surface EMG examination ${ }^{(24)}$.

The goal of any prosthetic rehabilitation is to provide a satisfactory prosthesis that allow good masticatory function. Thus, factors that may affect the masticatory muscle activity are worth investigation. This study was designed to evaluate and compare the effect of CAD/CAM as construction method for complete denture on the activity of Masseter and anterior temporalis muscles with the effect of the conventional method of complete denture construction.

According to the findings of the present study, regarding masseter muscle activity, the hypothesis that there is a difference in muscle activity between the study groups was accepted. Complete dentures constructed by CAD/CAM technology produced significantly higher EMG masseter muscle activity compared with EMG masseter muscle activity for complete dentures constructed by conventional technique after one month of dentures use. Likewise, the anterior temporalis muscle activity, the hypothesis that there is a difference in muscle activity between the study groups was accepted as well. Complete dentures constructed by $\mathrm{CAD} /$ CAM technology produced significantly higher EMG anterior temporalis muscle activity compared with EMG anterior temporalis muscle activity for complete dentures constructed by conventional technique after one month of dentures use.

Up to date there are no clinical studies have been found evaluating the influence on EMG muscles activity in edentulous patients treated with CAD/CAM versus conventional complete denture. Considering that, the results of this study cannot be compared with those of another similar study.

The results of this study is most likely attributed to suspected improved retention and stability of the complete denture constructed by $\mathrm{CAD} / \mathrm{CAM}$ technology if compared with the conventional technique of denture processing ${ }^{(12,13,25,26)}$. In a clinical case study conducted by Ali and Al-Harbi (13) the retention of maxillary complete denture was evaluated for both conventional and $\mathrm{CAD} / \mathrm{CAM}$ constructing techniques, maxillary $\mathrm{CAD} / \mathrm{CAM}$ denture base retention was increased as compared to that of conventionally constructed denture. The authors in this clinical case study attributed 
the significant improvement in denture retention, stability as well as clinical performance to good adaptation and border seal of CAD/CAM complete denture.

In another prospective clinical study done by Mathew et al ${ }^{(25)}$, they evaluated and compared treatment outcomes in digital and conventional complete removable dental prosthesis. Data analysis revealed significantly higher ratings for the digital complete denture relative to the denture base contour, fit, extension, stability and retention. As mentioned by Mathew et al ${ }^{(25)}$ this findings could be explained by improved fit due to the absence of polymerization shrinkage and the unique method of milling the digital prosthesis from a pre-polymerized block of acrylic resin.

In 2017 AlHelal et al (26), compared the retention of the maxillary complete denture bases constructed by CAD/CAM (AvaDent) with those of conventional heat-polymerized denture bases. The results revealed that, the retention offered by milled complete denture bases from pre-polymerized (Polymethyl Methacrylate) resin was significantly higher than that offered by conventional heat polymerized denture bases. This is most likely as the lack of polymerization shrinkage associated with $\mathrm{CAD} / \mathrm{CAM}$ denture bases results in an improved fit, and subsequent improving retention.

Furthermore, an in-vitro study was conducted by Steinmassl et al ${ }^{(12)}$ to test the assumption of higher CAD/CAM denture base fit than the conventionally constructed denture bases. They concluded that $\mathrm{CAD} / \mathrm{CAM}$ constructed denture has better fit than conventionally constructed one.

There is a direct positive correlation between complete denture retention and improved fit and the masticatory muscle activity recorded by EMG, this postulation could be supported by previous studies conducted to evaluate the effect of improved complete denture retention, fit and /or stability on the masticatory muscle activity ${ }^{(27-29)}$.
It should be acknowledged that there are limitations to this study, and the results may not be applicable to all edentulous patients. The followup period after denture insertion and EMG activity record was limited (30 days). Also, only male patients were participate in this study. Further research that considers these limitations and larger sample size is recommended to confirm findings.

\section{CONCLUSION}

By taking the limitations of this study into consideration, it can be concluded that $\mathrm{CAD} /$ CAM constructed denture has positive impact on the masticatory muscle activity if compared with conventionally constructed denture.

\section{REFERENCES}

1. Xie Q, Ding T, Yang G. Rehabilitation of oral function with removable dentures - still an option? J Oral Rehabil 2015; 42:234- 242.

2. Neto A F, Mestriner J W and Carreiro A F Masticatory efficiency in denture wearers' withbilateral balanced occlusion and canine guidance. Braz Dent J 2010; 21: 165-9.

3. Van der Bilt A. Assessment of mastication with implications for oral rehabilitation: A review. J Oral Rehabil 2011; 38: 754-80.

4. Tallgren A, Lang BR, Holden S, Miller RL. Longitudinal electromyographic study of swallowing patterns in completedenture wearers. Int J Prosthodont 1995; 8: 467-78.

5. Pruzansky S. The application of electromyography to dental research. J Am Dent Assoc 1952; 44: 49-68.

6. Cunha TR, Della Vecchia MP, Regis RR, et al. A randomised trial on simplified and conventional methods for complete denture fabrication: masticatory performance and ability. J Dentistry 2013; 41:133-42.

7. Goodacre CJ, Garbacea A, Naylor WP, Daher T, Marchack CB, Lowry J. CAD/CAM fabricated complete dentures: concepts and clinical methods of obtaining required morphological data. J Prosthet Dent 2012;107(1):34-46.

8. Kattadiyil MT, Jekki R, Goodacre CJ, Baba NZ. Comparison of treatment outcomes in digital and conventional complete removable dental prosthesis fabrications in a predoctoral setting. J Prosthet Dent 2015; 114(6):818-825. 
9. Infante L, Yilmaz B, McGlumphy E, Finger I. Fabricating complete dentures with CAD/CAM technology. J Prosthet Dent 2014; 111(5):351-5.

10. Han W, Li Y, Zhang Y, Lv Y, Zhang Y, Hu P, Liu H, Ma Z, Shen Y. Design and fabrication of complete dentures using CAD/CAM technology. Medicine (Baltimore). 2017; 96(1):e5435.

11. Li Y, Han W, Cao J, Iv Y, Zhang Y, Han Y, Shen Y, Ma Z, Liu H. Design of Complete Dentures by Adopting CAD Developed for Fixed Prostheses. J Prosthodont. 2018 Feb; 27(2):212-219.

12. Steinmassl O, Dumfahrt H, Grunert I, Steinmassl PA. $\mathrm{CAD} / \mathrm{CAM}$ produces dentures with improved fit. Clin Oral Investig 2018 Nov; 22(8):2829-2835.

13. Ali MSA, Al-Harbi FA. Posterior palatal seal area established in conventional and CAD/CAM fabricated complete denture techniques: Clinical Case Study. J Den Craniofac Res 2016; 1:1-6.

14. Ogden A. BSSPD Guidelines in Prosthetic and Implant Dentistry. London: Quintessence Publishing Co. Ltd. for the British Society for the Study of Prosthetic Dentistry 1996. p. 7-11.

15. Ferrario VF, Tartaglia GM, Galletta A, Grassi GP, Sforza C. The influence of occlusion on jaw and neck muscle activity: a surface EMG study in healthy young adults. J Oral Rehabil 2006; 33:341-48.

16. Henriksson KL. Distribution, number and size of different types of fibres in whole cross-sections of female $\mathrm{m}$ tibialis anterior. An enzyme histochemical study. Acta Physiol Scand 1985; 123:229-35.

17. Bingefors K, Isaccon D. Epidemiology, com-morbidity and impact on health-related quality of life of self-reported headache and musculoskeletal pain- a gender perspective. Eur J Pain 2004; 8:435-50.

18. Kuba T, Quirones-Jenab V. The role of female gonadal hormones in behavioral sex difference in persistent and chronic pain: clinical versus preclinical studies. Brain Res Bulletin 2005; 66:179-88.
19. Fillingim RB, Maixner W. The influence of resting blood pressure and gender on pain responses. Psychosomatic Medicine 1996; 58:326-32.

20. Logan HL, Gedney JJ. Sex differences in the long-term stability of fore head cold pressor pain. J Pain 2004; 5:406-12.

21. Von Gonten AS, Palik JE, Oberlader BA and Rugh JD. Nocturnal electromyographic evaluation of masseter muscle activity in the complete denture patient. J Prosthet Dent 1986; 56:624-9.

22. Henrikson T, Ekberg EC, Nilner M. Masticatory efficiency and ability in relation to occlusion and mandibular dysfunction in girls. Int J Prosthodont 1998;11:125-32.

23. Mathew TK, Charles JG, Nadim ZB. CAD/CAM complete dentures: a review of two commercial fabrication systems. J Calif Dent Assoc. 2013 Jun; 41(6): 407-416.

24. Castroflorio T, Bracco P, Farina D. Surface electromyography in the assessment of jaw elevator muscles. J Oral Rehabil 2008 Aug; 35(8): 638-45

25. Kattadiyil MT, Jekki R, Goodacre CJ, Baba NZ. Comparison of treatment outcomes in digital and conventional complete removable dental prosthesis fabrications in a predoctoral setting. J Prosthet Dent 2015; 114:818-25.

26. AlHelal A, AlRumaih HS, Kattadiyil MT, Baba NZ, Goodacre CJ. Comparison of retention between maxillary milled and conventional denture bases: a clinical study. J Prosthet Dent 2017; 117(2):233-238.

27. Van Kampen F MC, Van der Bilt A, Cune S and bosman F. The influence of various attachment types in mandibular implant-retained overdentures on maximum bite force and EMG. J Dent Res 2002; 81 (3):170-173.

28. Caloss R, Al-Arab M, Finn RA. Throckmorton GS. The effect of denture stability on bite force and muscular effort. J Oral Rehabil 2001; 38 (6):434- 439.

29. Rastogi A, Srivastava S, Gaur A, Dupare A, Rastogi S, Kamatagi L. Electromyographic Evaluation of the Effect of Lined Dentures on Masticatory Muscle Activity in Edentulous Subjects. J Clin Diagn Res. 2015 Aug; 9(8):ZC80-3. 